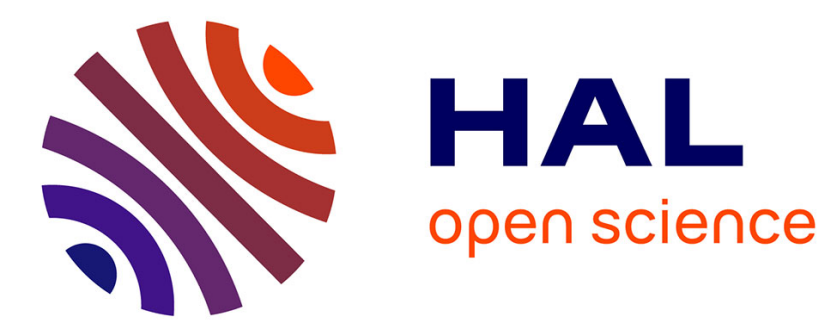

\title{
Tailoring the absorption in a photonic crystal membrane: A modal approach
}

Romain Peretti, Guillaume Gomard, Christian Seassal, Xavier Letartre, Emmanuel Drouard

\section{To cite this version:}

Romain Peretti, Guillaume Gomard, Christian Seassal, Xavier Letartre, Emmanuel Drouard. Tailoring the absorption in a photonic crystal membrane: A modal approach. Photonics Europe, Apr 2012, Brusselles, Belgium. pp.84250Q, 10.1117/12.922427 . hal-00691540

\section{HAL Id: hal-00691540 \\ https://hal.science/hal-00691540}

Submitted on 27 Apr 2012

HAL is a multi-disciplinary open access archive for the deposit and dissemination of scientific research documents, whether they are published or not. The documents may come from teaching and research institutions in France or abroad, or from public or private research centers.
L'archive ouverte pluridisciplinaire HAL, est destinée au dépôt et à la diffusion de documents scientifiques de niveau recherche, publiés ou non, émanant des établissements d'enseignement et de recherche français ou étrangers, des laboratoires publics ou privés. 


\title{
Tailoring the absorption in a photonic crystal membrane: A modal approach
}

\author{
Romain Peretti*a $^{*}$, Guillaume Gomard ${ }^{\mathrm{a}}$, Christian Seassal ${ }^{\mathrm{a}}$, Xavier Letartre ${ }^{\mathrm{a}}$, Emmanuel Drouard ${ }^{\mathrm{a}}$ \\ ${ }^{a}$ Université de Lyon, Institut des Nanotechnologies de Lyon (INL), UMR 5270, CNRS-INSA-ECL- \\ UCBL, France \\ Ecole Centrale de Lyon, 36 Avenue Guy de Collongue, 69134 Ecully Cedex, France
}

\begin{abstract}
In order to achieve high efficiency photovoltaic devices and sensors, we propose to implement photonic crystals on thin absorbing layers in such a way to generate two Bloch mode resonances with opposite symmetries. Through FDTD and RCWA simulations, we track and adjust the characteristics of these modes so as to reach their degeneracy. Design and simulations were carried out considering a hydrogenated amorphous silicon layer. We demonstrate that up to $92 \%$ absorption can be achieved, far above the $50 \%$ limit corresponding to the critical coupling condition between an incident wave and an optical resonance. Moreover, the robustness of the absorption peak was tested by varying both the topographical parameters of the $\mathrm{PhC}$ membrane and the angle of incidence. Finally, some guidelines are provided to generalize our approach for the design of broadband absorbers.
\end{abstract}

Keywords: Photonic crystal, absorption enhancement, photovoltaic solar cell, indoor solar cell, sensor

\section{INTRODUCTION}

Achieving a maximal incident light absorption in a given medium and for a specific spectral range is of prime importance for solar [1] and indoor [2] photovoltaic cells or for sensors [3]. Parallel to this, absorption in a reduced volume, and in particular in the layers of thin film solar cells, are preferred so as to decrease the costs and to facilitate the integration of those devices, among others. Unfortunately, the single pass absorption of light in thin layers is too low for a proper operation, especially at wavelengths close to the material bandgap. Indeed, in this spectral domain, the extinction coefficient (denoted $\kappa$ ) can be low, whereas the reflectance may remain high due to a large index contrast with the surrounding medium. So as to tackle these two issues simultaneously, resonant optical modes can be used to enhance the absorption $[1,4]$ by increasing the optical path length in the absorbing layer while limiting the reflectance. In this paper, we particularly focus on Bloch mode resonances generated in layers patterned as planar Photonic Crystals (PhC); these structures will further be referred to as photonic membranes.

In the following, we first introduce the parameters of an absorbing photonic membrane and its corresponding band diagram, from which two orthogonal modes are extracted to conduct the study. The critical coupling conditions of an isolated mode are then analyzed in terms of surface filling factor. In a second step, the PhC parameters are optimized, which enables to fulfill the necessary conditions for the creation of a degeneracy mode exhibiting a high absorption, close to $100 \%$. The last section of this communication aims at evaluating the potential of this concept to be integrated in a real device by looking at its robustness with regards to the $\mathrm{PhC}$ parameters and the angle on incidence.

\section{METHODOLOGY}

\subsection{Description of the absorbing patterned membrane}

The configuration studied, depicted on the left-hand side of Figure 1, is a $\mathrm{PhC}$ membrane with a $C_{4}$ symmetry, a structure capable of enhancing the absorption [5] and that can be easily fabricated by various methods including holographic [6], e-beam [7] or nano-imprint [8] lithography.

Photonic Crystal Materials and Devices X, edited by Hernán Ruy Míguez, Sergei G. Romanov,

Lucio Claudio Andreani, Christian Seassal, Proc. of SPIE Vol. 8425, 84250Q · C 2012 SPIE

CCC code: $0277-786 X / 12 / \$ 18 \cdot$ doi: $10.1117 / 12.922427$

Proc. of SPIE Vol. 842584250 Q-1 

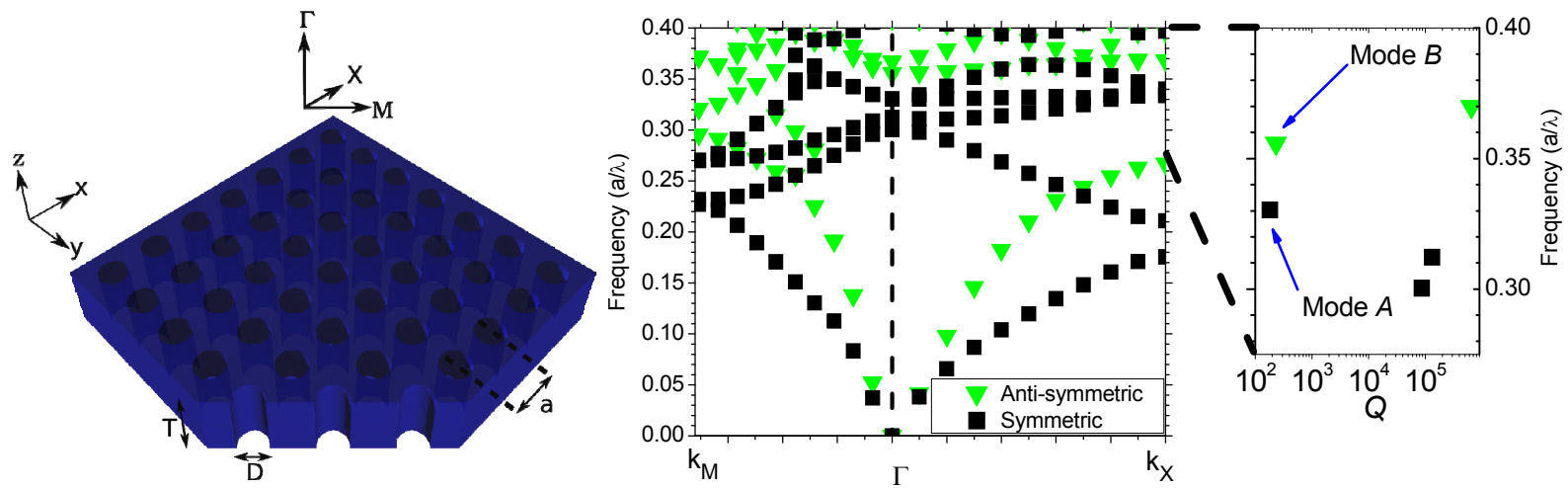

Figure 1. Left: Schematic view of the 2D PhC membrane with its corresponding parameters. Center: Band diagram of this membrane with $T=a / 2$ and $f f=15 \%$ for both the vertically symmetric (black squares) and anti-symmetric (green triangles) modes. Right: Frequency versus the quality factors of the modes in $\Gamma$, zoomed in the frequency range of interest

The material refractive index is set to $n=4.06$ and its extinction coefficient is fixed to $k=10^{-2}$, which corresponds roughly to the optical indices of amorphous silicon (a-Si) at $720 \mathrm{~nm}$ [9]. In the remainder of this paper, the thickness of the membrane will be referred to as $T$, the lattice parameter as $a$ and the air surface filling factor as $\mathrm{ff}$. The band diagram of the structure reported on Figure 1 was calculated by the Finite Difference Time Domain (FDTD) method and using Harminv [10] in order to illustrate the modal behavior of the absorbing membrane. Moreover, the vertical symmetry (related to the guided mode order) is shown by a specific color to highlight the orthogonality of the modes involved (symmetric and anti-symmetric modes will be orthogonal). It can be noticed that only two modes (denoted $A$ and $B$ ) exhibit quality factor $(Q)$ that is low enough to be couple to propagative waves. In other words, only those two modes will participate significantly to the absorption in the frequency range of interest.

The starting point of this study is the concept of additive absorption between two modes. For this purpose, the Rigorous Coupled Wave Analysis (RCWA) method available in CAMFR-GARCLED [11] was used to compute the photonic properties of the studied structure. Preliminary convergence tests were carried out to assess the error on the absorption values which was estimated to be around $5 \%$ in the case of a single absorption peak. In addition, all the simulations presented below were made on a non-dispersive material and, unless specified, under a normal incidence.

\subsection{Modal analysis}

To go further, we focused on a spectral region containing the two orthogonal modes $A$ and $B$. Their electric field calculated by RCWA is reported for a cross-section view of the membrane on Figure 2.

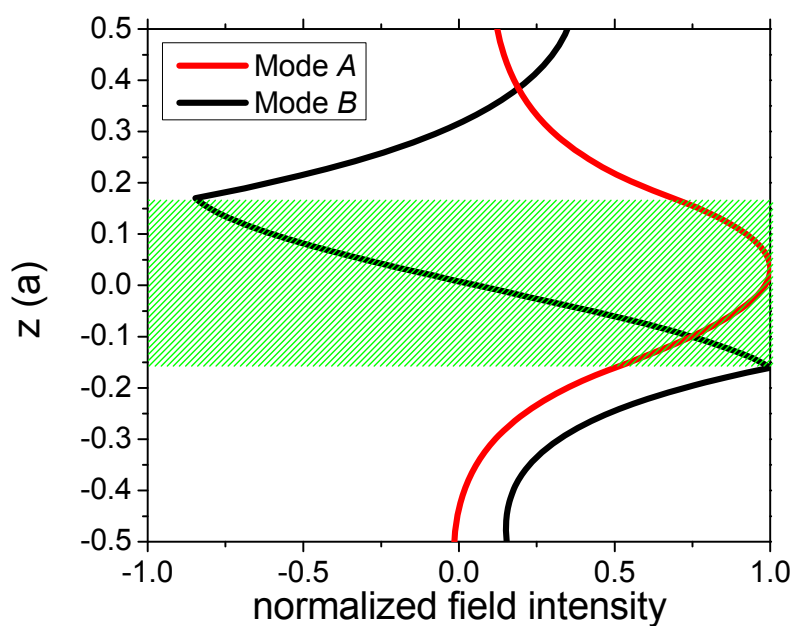

Figure 2. Normalized electric field intensity for mode $A$ (in red) and mode $B$ (in black) viewed across the membrane.

The latter is represented by the green dashed area 
Those profiles clearly highlight the fact that mode $A$ is symmetric and mode $B$ is anti-symmetric in the vertical direction, even if one can see that the symmetry (or the anti-symmetry) is not perfect because of the plane wave excitation from the front side (positive $\mathrm{z}$ coordinates). Besides, if the membrane is divided into two regions whose vertical limit is set at $\mathrm{z}=0$, it can be noticed that the overlap integral of the two modes in the first region is the opposite of the one in the second region. This leads to a null global overlap integral, which can be accounted for by the orthogonality of modes $A$ and $B$. To summarize, those two modes are good candidates to apply the theory developed in [12] in order to reach the highest absorption possible for a given wavelength, which will be the point of the next sections.

\section{RESULTS AND ANALYSIS}

\subsection{Single mode absorption optimization}

First of all, the absorption of the photonic membrane should be optimized for each single mode. This is achieved in critical coupling conditions for a particular value of $Q$. One topographical parameter which enables to easily impact the quality factor of a mode is the surface filling factor. Indeed, increasing the hole diameter strengthens the coupling between the incoming plane wave and a guided mode, which in the end lowers $Q$. Figure 3 presents the resulting absorption of the $\mathrm{PhC}$ for mode $A$ by varying $f f$ from $0 \%$ (unpatterned slab) to $30 \%$, together with the $Q$ values on the top axis.

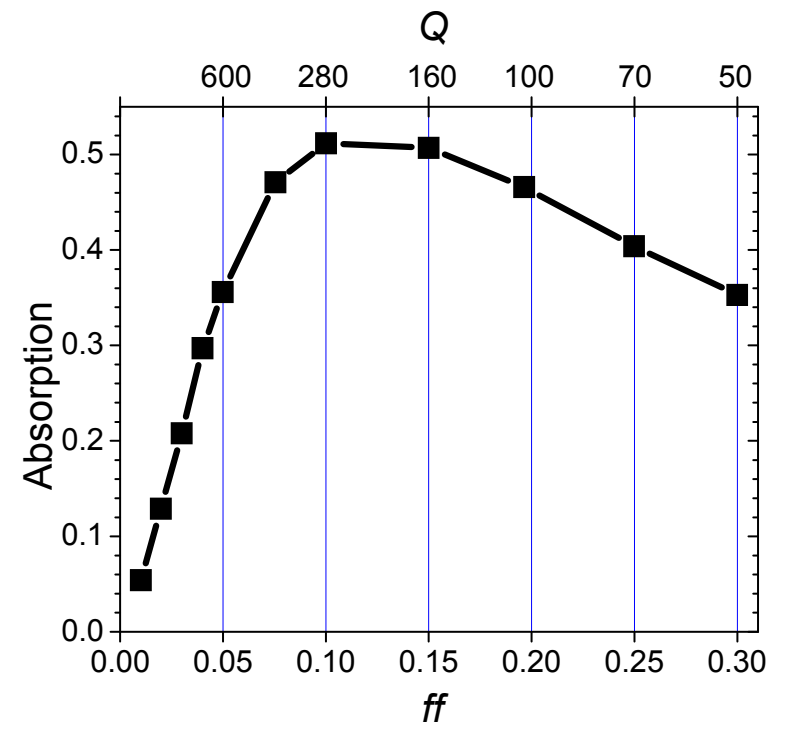

Figure 3. Absorption of mode $A$ as a function of the surface filling factor in air for $T=0.35 a$. The top axis shows the $Q$ of the mode for the corresponding $f f$

This figure exhibits the usual behavior for critical coupling, namely an optimal $Q$ (here around 200) when the external losses (coupling) equal the absorption losses. As predicted by the TDCMT, for this particular value of $Q$, the absorption is limited to $50 \%$. A similar behavior could be observed for mode $B$ but is not shown here.

\subsection{Dual modes absorption optimization}

To overcome the $50 \%$ absorption limit, the use of several orthogonal modes is now required. Since modes $A$ and $B$ are not supported by the same guided mode orders (order 0 for mode $A$ and order 1 for mode $B$ ), the effective index and therefore the dispersion of their resonant frequency will be different with regards to the membrane thickness $T$. This enables to achieve degeneracy by tuning $T$ so that the resonant frequency of both modes is set to the same value. Furthermore, $f f$ and $T$ should be optimized not only to get the degeneracy state but also to maximize the absorption in each part (symmetric part for mode $A$ and anti-symmetric part for mode $B$ ) of the degenerated mode. This was performed by combining RCWA simulations to a conjugate gradient method, resulting in $f f_{\text {optim }}=20.7 \%$ and $T_{\text {optim }}=0.59 a$. The absorption spectrum corresponding to this optimized configuration is reported in Figure 4, as well as the ones involving thicknesses below $(T=0.33 a)$ and above $(T=0.85 a) T_{\text {optim }}$. 


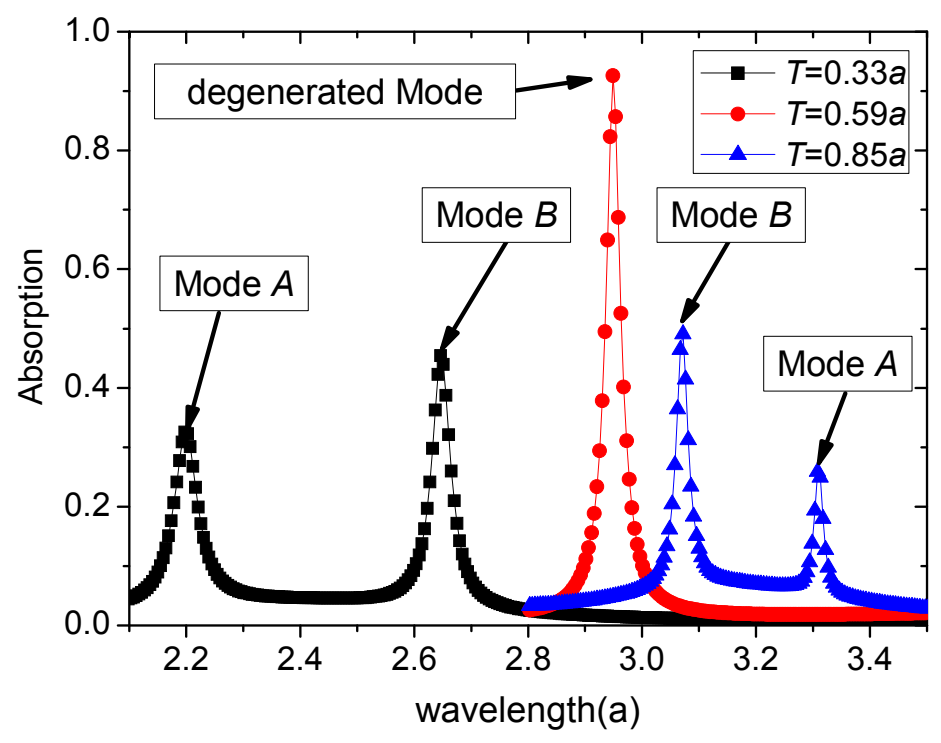

Figure 4. Absorption spectra of the patterned membrane for $f f=20.7 \%$ and $T=0.33 a, 0.59 a$ and $0.85 a$ around the absorption peaks of interest

On this figure, it can be verified that the usually admitted $50 \%$ limit for a symmetric and isolated membrane $[13,14,4]$ is respected when the modes are not degenerated $(T=0.33 a$ and $T=0.85 a)$ but is significantly overcome for the degenerated mode, for which the absorption is about $92 \%$. When increasing the thickness of the membrane, the dispersion characteristic of the modes makes the resonant frequencies of modes $A$ and $B$ crossing only once, so that the degeneracy can only be obtained for a given thickness. Eventually, it is noteworthy that the width of the degeneracy peak corresponds to the width of the two isolated peaks which are related to the absorption of the material through the critical coupling conditions [4].

The example above is one of the highlight of our study since it demonstrates how absorptions as high as $92 \%$ can be obtained thanks to an appropriate modal engineering of the patterned membrane. However, to apply this concept to real devices, it is of particular importance to evaluate the variation of this absorption as a function of the membrane parameters which can be different from the optimized ones due to technological uncertainties. This point was investigated in Figure 5 by varying $f f$ and $T$ around the optimized configuration. 


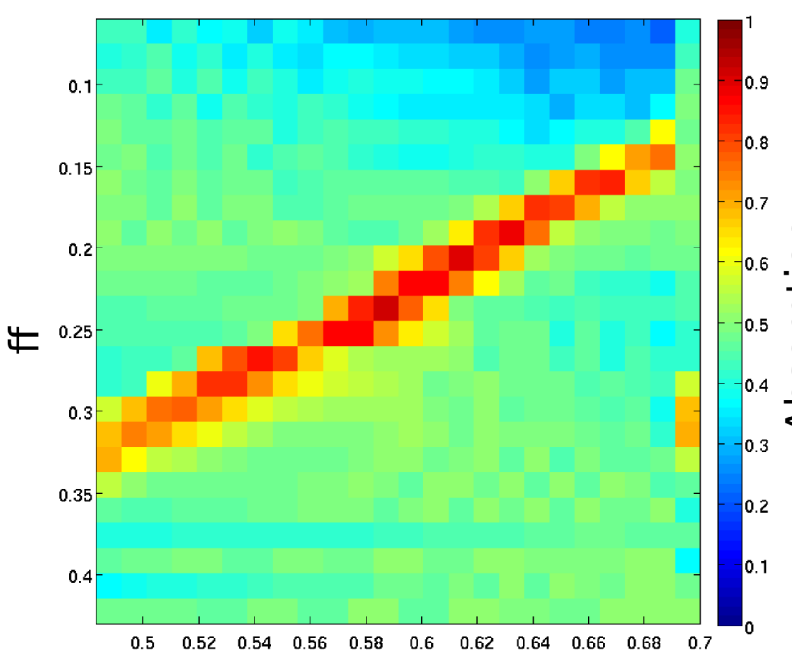

Thickness (a)

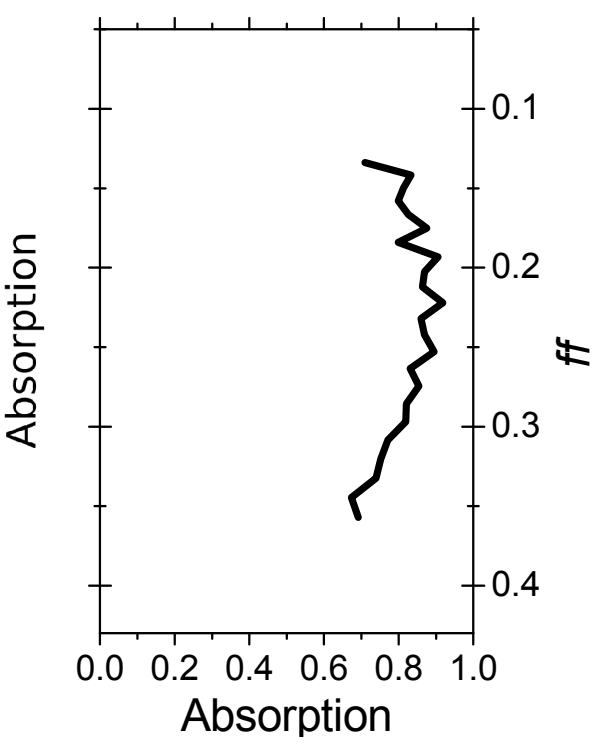

Figure 5. Left: Mapping of the absorption around the optimized degenerated mode as a function of $f f$ and T. Right: Cross-section of the mapping along the "degeneracy axis".

One can see on this figure that the robustness of the parameters to keep the degeneracy and to stay close to the critical coupling conditions is about $2 \%$ for $T$ and $1 \%$ for $f f$. Nevertheless, the degeneracy is achieved for several couples of parameters distributed along a characteristic axis on Figure 5. When moving away from the optimized configuration, the absorption gradually decreases since the critical coupling tends to disappear. The right part of Figure 5 reports the absorption along this axis, which remains almost always higher than $70 \%$ for the values considered. This means that the degeneracy can be obtained for a large range of thicknesses after adjusting $f f$ properly.

If the degeneracy peak absorption value is an important attribute, the dispersion of this peak with $f f$ and $T$ is also a key point and is consequently represented on.Figure 6
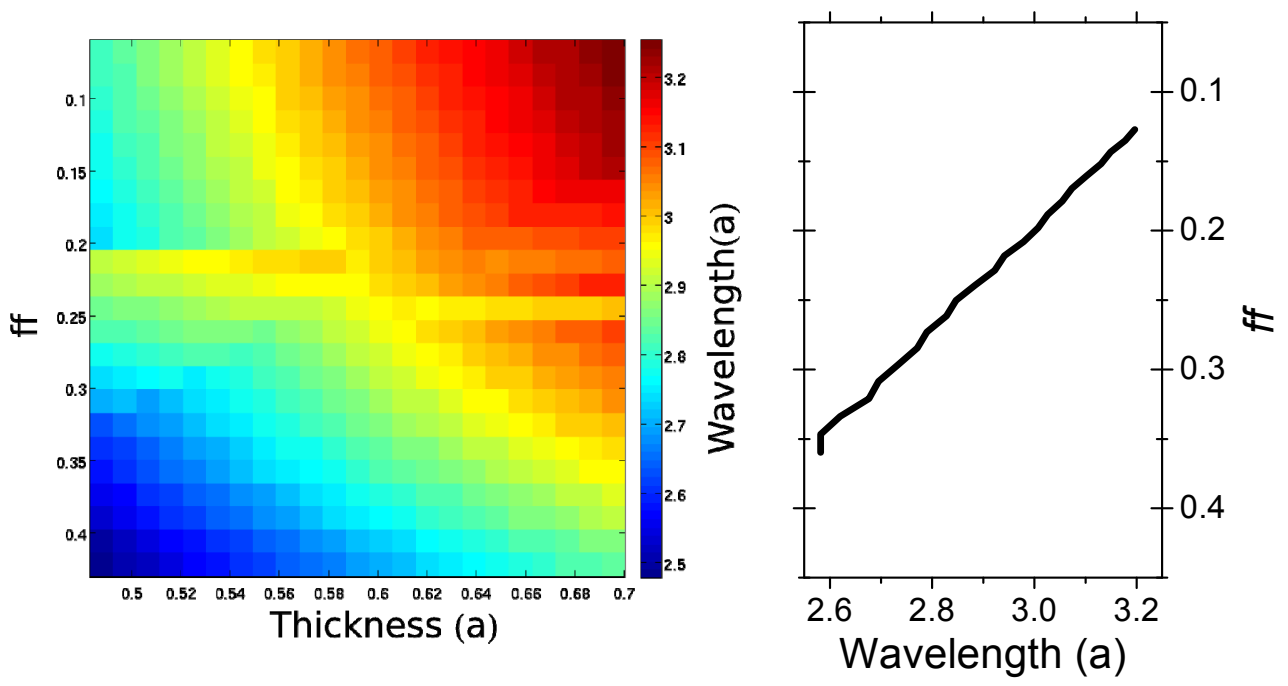

Figure 6. Left: Mapping of the peak wavelength as a function of $f f$ and T. Right: Cross-section of the mapping along the "degeneracy axis".

It can be checked that the peak absorption wavelength increases with both $f f$ and $T$ providing another way of tuning it apart from a scaling of the membrane. 
Thus, it was shown that the degeneracy is not restricted to a single couple of parameters but can be reached for numerous $(f f, T)$ values. To extend the concept to the case of an absorption resulting from a collection of modes (E.g for photovoltaic applications), one can conclude that provided that the modal density is high enough, several degeneracy will be created in a given spectral range, which can lead to a broadband absorption enhancement. In addition, it should be stressed that the dispersion of the modal properties will not be identical in that case. However, as some degenerated modes will be lost, others will appear enabling the absorption to remain high and more robust than in the case of a single degenerated mode.

\subsection{Influence of the angle of incidence on the absorption of the degenerated state}

The tolerance of the absorption towards the angle of incidence of the incoming light is another point which needs to be addressed with a view to integrate this concept in operating devices. The absorption spectra for the degenerated and optimized configuration are shown for angles of incidence $\alpha$ increasing from 0 to $15^{\circ}$ (See Figure 7).

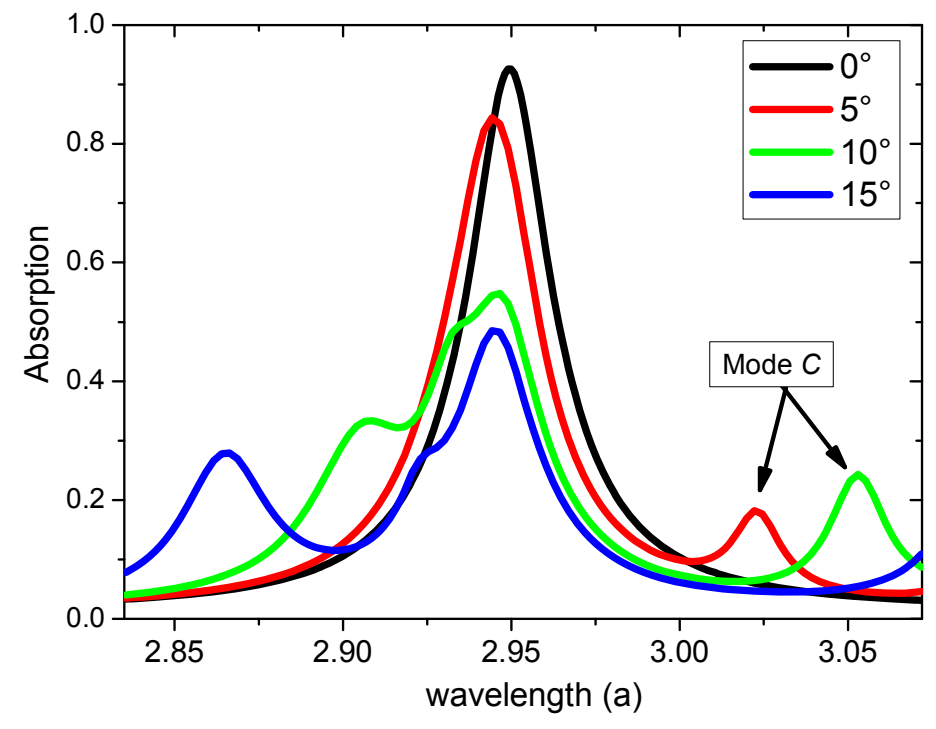

Figure 7: Influence of the angle of incidence on the absorption spectrum of the optimized degenerated peak

Below $\alpha=5^{\circ}$, the absorption peak is only slightly modified and absorption still reaches more than $80 \%$. For larger angles of incidence, it can be noticed that the main absorption peak splits into two lower peaks $(<50 \%$ in absorption) as the degeneracy is lost. Moreover, for non-normal incidence, a new mode appears (denoted mode $C$ in Figure 7) which is redshifted when $\alpha$ is increased. To go further in the study of modes $A, B$ and $C$, the mapping of their electric field intensity is reported in Figure 8. 
Mode A

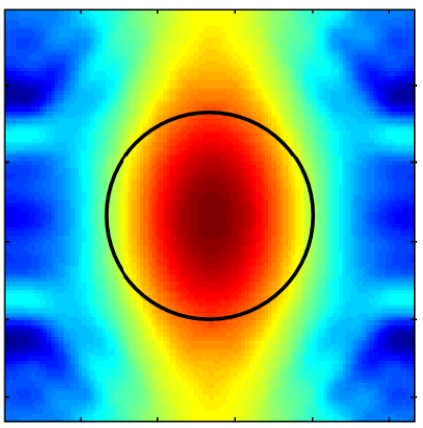

Mode B

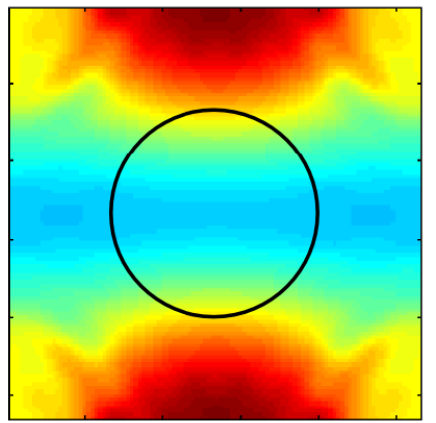

Mode C

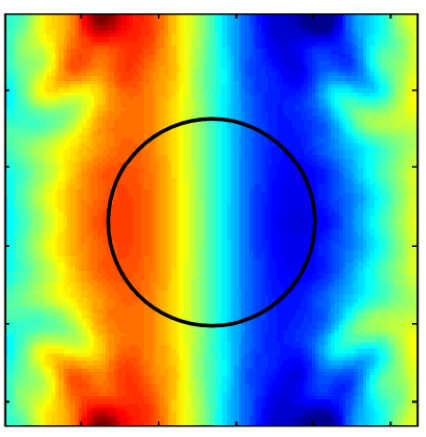

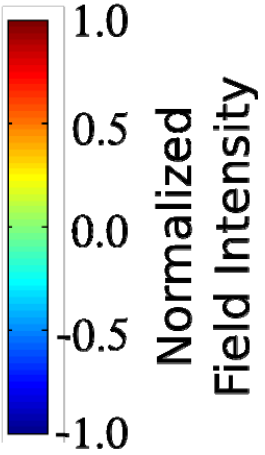

Figure 8. Mapping of the normalized field intensity for mode $A$ (left), mode $B$ (center) and mode $C$ (right) over one period in the $\mathrm{x}$ and $\mathrm{y}$ directions. The air hole is centered and depicted as a circle

It can be seen that modes $A$ and $B$ do not exhibit any anti-symmetrical field intensity distribution, that is why they can couple to the incoming light under normal incidence. On the contrary, mode $C$ is characterized by an anti-symmetry axis in the middle of the elementary cell, which forbids any coupling under normal incidence due to a null overlap integral. Thus, it can only absorb light when $\alpha>0^{\circ}$, as depicted in Figure 7. In the case of a collection of modes, the angle of incidence will change the wavelength of each mode according to their own dispersion. Again, if the density of mode is high enough, the lost of some degeneracy can be counterbalanced by the creation of new ones resulting in a robust behavior towards $\alpha$.

\section{CONCLUSION}

In this paper, we optimized the absorption in a 2D PhC membrane at a given wavelength using one symmetric and one anti-symmetric mode with the Rigorous Coupled Wave Analysis method. Those two modes of opposite symmetry along the thickness of the membrane were chosen because they do couple with light under normal incidence. The $\mathrm{PhC}$ parameters $(f f$ and $T)$ were optimized leading to a maximal absorption of $92 \%$. Actually in a recent paper [12] it was shown using the Time Domain Coupled Mode Theory (TDCMT) that an absorption up to $100 \%$ could be obtained in a symmetric and isolated membrane overcoming the usually admitted limit of $50 \%[15,14]$. This could be achieved by combining two modes in their critical coupling conditions to create a degenerated state. In the present case, the resulting absorption of $92 \%$ could still be improved if those two conditions were met. In order to adopt a point of view closer to potential applications, we assessed the robustness of the concept with regards to the topographical parameters and the angle of incidence. It was concluded that with a good technological precision, absorptions higher than $90 \%$ could be reached, while absorption above $70 \%$ was still accessible with reasonable technological uncertainties. Moreover, the degenerated mode is robust for angles of incidence ranging up to $5^{\circ}$. Thus, this concept can directly be applied to detection or narrow-band emitting devices in order to control the spectral bandwidth and/or their directivity.

To extend this concept to broadband absorbers such as indoor or solar photovoltaic cells, a collection of modes have to be used $[16,13]$. Since the width of the absorption peak is directly related to material absorption, the spectral density of the peaks needed around one targeted wavelength $\lambda$ can be deduced directly from the value of the extinction coefficient of the material. As a matter of fact, given a $\Delta \lambda$ range around $\lambda$, the order of magnitude of modes spectral density needed to optimize the absorption at $100 \%$ is about $n \kappa^{-1} \Delta \lambda / \lambda$. In such a configuration, if the symmetric and antisymmetric modes are equally populated, the degeneracy will be obtained naturally due to the spectral overlap of the modes as was observed for example in [9]. In addition, as it can be seen on the band diagram of the patterned membrane, the flatness of the band around the $\Gamma$ point is low enough to keep most of the modes in the same spectral regions, leading to an optically robust device.

\section{ACKNOWLEDGMENT}

This work was partly supported by France Telecom, Orange Labs and by the Region Rhône-Alpes. 


\section{REFERENCES}

[1] Mallick, S., Sergeant, N., Agrawal, M., Lee, J., and Peumans, P., "Coherent light trapping in thinfilm photovoltaics," MRS BULLETIN 36, 453-460 (2011).

[2] Reich, N., van Sark, W., and Turkenburg, W., "Charge yield potential of indoor-operated solar cells incorporated into product integrated photovoltaic (pipv)," Renewable Energy 36(2), 642 647 (2011).

[3] Lai, W.-C., Chakravarty, S., Wang, X., Lin, C., and Chen, R. T., "Photonic crystal slot waveguide absorption spectrometer for on-chip near-infrared spectroscopy of xylene in water," Applied Physics Letter 98(2), 023304 (2011).

[4] Park, Y., Drouard, E., Daif, O. E., Letartre, X., Viktorovitch, P., Fave, A., Kaminski, A., Lemiti, M., and Seassal, C., "Absorption enhancement using photonic crystals for silicon thin film solar cells," Opt. Express 17, 14312-14321 (2009).

[5] Gomard, G., Meng, X., Drouard, E., Hajjam, K. E., Gerelli, E., Peretti, R., Fave, A., Orobtchouk, R., Lemiti, M., and Seassal, C., "Light harvesting by planar photonic crystals in solar cells: the case of amorphous silicon," Journal of Optics 14(2), 024011 (2012).

[6] Meng, X., Gomard, G., Daif, O. E., Drouard, E., Orobtchouk, R., Kaminski, A., Fave, A., Lemiti, M., Abramov, A., i Cabarrocas, P. R., and Seassal, C., "Absorbing photonic crystals for silicon thin-film solar cells: Design, fabrication and experimental investigation," Solar Energy Materials and Solar Cells 95, Supplement 1(0), S32 - S38 (2011).

[7] Utkin, D. and Nasimov, D., "Formation of two-dimensional photonic crystals by electron-beam lithography," International Conference and Seminar of Young Specialists on Micro/Nanotechnologies and Electron Devices (EDM), 2011 -, 119-121 (2011).

[8] Chen, J. and Sun, K., "Enhancement of the light conversion efficiency of silicon solar cells by using nanoimprint anti-reflection layer," Solar Energy Materials and Solar Cells 94(3), 629 - 633 (2010).

[9] Gomard, G., Drouard, E., Letartre, X., Meng, X., Kaminski, A., Fave, A., Lemiti, M., Garcia-Caurel, E., and Seassal, C., "Two-dimensional photonic crystal for absorption enhancement in hydrogenated amorphous silicon thin film solar cells," Journal of Applied Physics 108(12), 123102 (2010).

[10]Mandelshtam, $\mathrm{V}_{.,}, \mathrm{Fdm}$ : the filter diagonalization method for data processing in $\mathrm{nmr}$ experiments," Progress in Nuclear Magnetic Resonance Spectroscopy 38(2), 159 (2001).

[11] http://camfr.sourceforge.net/.

[12]Peretti, R., Gomard, G., Seassal, C., Letartre, X., and Drouard, E., "Modal approach for tailoring the absorption in a photonic crystal membranmodal approach for tailoring the absorption in a photonic crystal membrane," Physical Review A Submited to, - (2012).

[13]Yu, Z., Raman, A., and Fan, S., "Fundamental limit of light trapping in grating structures," Opt. Express 18, A366-A380 (2010).

[14]Chutinan, A. and John, S., "Light trapping and absorption optimization in certain thin-film photonic crystal architectures," Phys. Rev. A 78, 023825 (2008). 
[15]Manolatou, C., Khan, M., Fan, S., Villeneuve, P., Haus, H., and Joannopoulos, J., "Coupling of modes analysis of resonant channel add-drop filters," Quantum Electronics, IEEE Journal of 35(9), 1322-1331 (1999).

[16]Yu, Z., Raman, A., and Fan, S., "Nanophotonic light-trapping theory for solar cells," Applied Physics A: Materials Science \& Processing 1, 1-11 (2010). 